\title{
Preface
}

Neuropsychobiology

Published online: March 4, 2019

\section{0th Biennial IPEG Meeting 2018 in Zürich}

\section{Electrophysiology in Neuropsychiatric Research: A Bright Future Ahead?}

Although electrophysiology is part of many clinical assessments, electroencephalogram (EEG) research especially in the neuropsychiatric field often faces criticism with some denying any substantial progress since the times of Hans Berger almost 100 years ago. Additionally, in the past decades, other, more visually attractive modalities such as functional MRI have been stealing the show from EEG research, attracting many young researchers. However, neither EEG nor functional MRI findings have made it into clinical routine to this day. So, it seems when looking ahead that prospects are not too promising, or are they?

One has to keep in mind that the EEG is a widely available, non-invasive approach to record data from neurons, which are the source of cortical activity. The EEG thereby provides a unique window into brain function at a millisecond resolution. This is the strength of EEG in all domains, from preclinical applications in animal research to diagnostics in humans. However, this also is a weakness, since the signals are so complex and rich in information that it is hard to understand the whole picture, and EEG researchers are still looking for some "ground truth." This is where the International Pharmaco EEG Society (IPEG) and its biennial meetings come into place: the IPEG provides a platform for exchanging new findings, discusses standards, and trains people in various fields of preclinical and clinical EEG research.

The future of personalized medicine relies on the ability to transfer preclinical findings from animal research and hypotheses from computational models to clinical applications. Additionally, objective information about individual electrophysiological profiles of the patient might help in the diagnostic process or when choosing the treatment that is most likely to help the patient. In the cooperation between the therapist and the patient, a shared decision-making process including the biomarker information might one day lead to a faster or more pronounced response by objectively choosing the best treatment.

The IPEG Meeting 2018 in Zürich again provided a great overview on cutting-edge neurophysiological research along the outlined track. Although it became clear that there is still a long way to go before electrophysiological applications will be part of an objective individualized approach in neuropsychiatry, the direction seems clear: multicenter studies and prospective randomized trials, testing standardized markers derived from animal research, and computational models or pilot studies followed by replication studies are urgently needed. Analysis 
should focus on shared data between groups, possibly also relying on new techniques such as deep learning. Also, research should not only differentiate between responders and nonresponders for treatment but should compare the probability of response for multiple treatments following a certain electrophysiological profile, including central and autonomous nervous system markers.
All these topics and even more were covered by the keynote speakers, the symposia, the free communications, and the poster presentations presented on the following pages.

Sebastian Olbrich

President of the IPEG 